\title{
NOUVELL
}

\section{Contrôle neural du remodelage osseux \\ Le rôle crucial de la leptine}

Karine Clément, Gérard Karsenty
> L'os contient deux types cellulaires, l'ostéoblaste, responsable de la formation osseuse, et l'ostéoclaste, responsable de sa résorption. La masse osseuse est maintenue grâce à un équilibre précis entre l'activité des ostéoclastes et celle des ostéoblastes. Cette fonction physiologique est appelée le remodelage osseux. L'ostéoporose est une maladie du remodelage osseux caractérisée par une augmentation de la résorption par rapport à la formation osseuse. La fréquence et la gravité de l'ostéoporose dans les pays développés expliquent l'effort de recherche pour élucider la régulation moléculaire de la masse osseuse. Le contrôle du remodelage osseux est complexe [1]. II fait intervenir des processus locaux avec une coopération entre les ostéoblastes et les ostéoclastes et un contrôle hormonal (Figure 1). Le laboratoire de Gérard Karsenty (Baylor College of Medicine, Texas, États-Unis) s'intéresse aux mécanismes moléculaires régulant la masse osseuse et a démontré que la leptine inhibe la formation osseuse [2]. Cette action requiert la liaison de la leptine à son récepteur hypothalamique. Le système nerveux sympathique sert de médiateur afférent à l'action de l'hormone sur le contrôle de la formation osseuse via le récepteur adrénergique $\beta 2$ ( $A D R \beta 2$ ), seul récepteur adrénergique exprimé dans l'ostéoblaste.

\section{La leptine favorise la résorption} osseuse par l'intermédiaire du système sympathique

Dans l'article publié dans Nature [3], il est montré que des souris, dont le gène codant pour le récepteur $A D R \beta 2$ a été invalidé, $A D R \beta 2^{-/-}$, ont une élévation des paramètres de formation de la masse osseuse. L'injection de leptine en intracérébroven- triculaire (ICV) ne diminue pas la formation osseuse, validant la nécessité d'une intégrité du système sympathique pour relayer les effets de la leptine sur l'os. Le phénotypage osseux des souris $A D R \beta 2^{-/-}$a révélé des surprises. Les paramètres de résorption osseuse étaient aussi diminués ; la baisse des cellules ostéoclastiques TRAP (tartrate resistant acid Nutrition, Inserm EA3502, Hôtel-Dieu, 1, place du Parvis de Notre-Dame, 75004 Paris, France.

G. Karsenty : Department of Molecular and Human Genetics, Baylor College of Medicine, One Baylor Plaza, Room S921, Houston, Texas 77030, États-Unis. karsenty@bcm.tmc.edu
K. Clément : Laboratoire de phosphatase) positives indique un défaut de différenciation oscléoclastique, et celle de la désoxypyridinoline (Dpd), un dysfonctionnement des ostéoblastes. Ces anomalies n'étaient pas corrigées par l'injection ICV de leptine. Les souris $A D R \beta 2^{-/-}$sont dans une situation idéale pour la prévention de l'ostéoporose: augmentation de la formation osseuse et baisse de sa résorption. L'analyse cellulaire et moléculaire de ce phénotype osseux a permis de montrer que le tonus sympathique favorise l'expression normale de RANKL

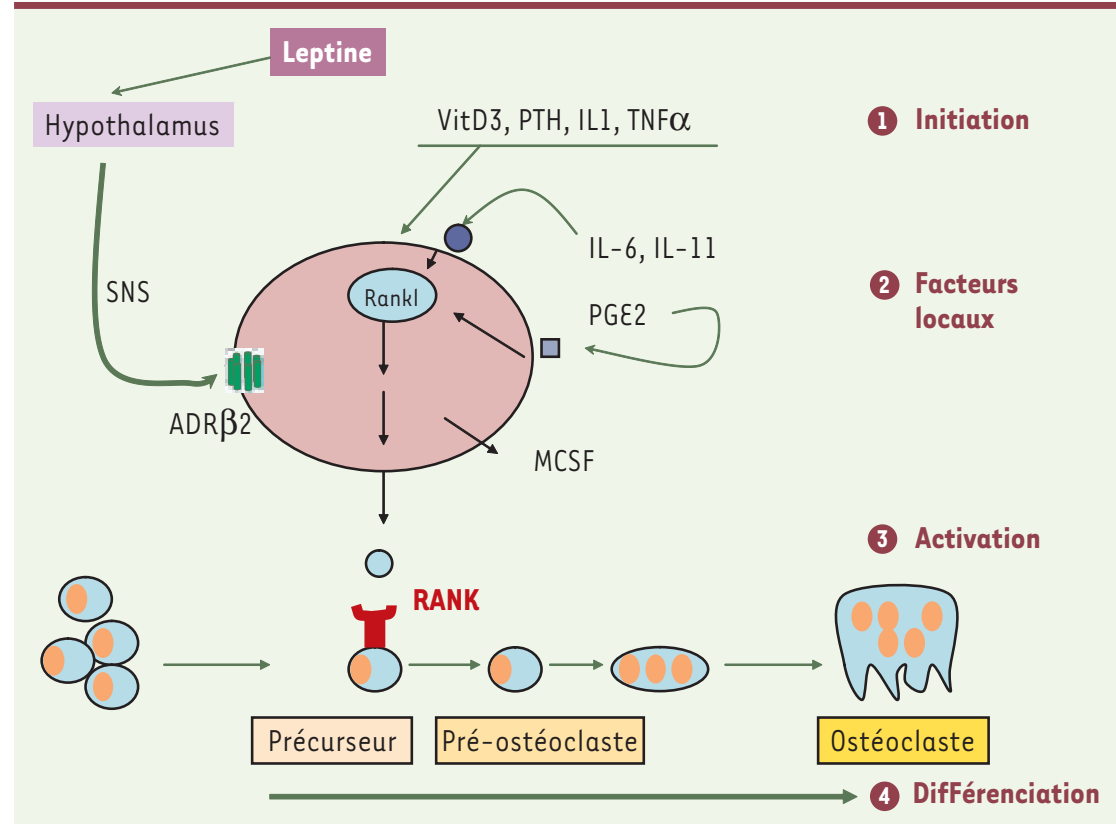

Figure 1. Facteurs locaux et hormones contrôlant la résorption osseuse. Classiquement, les hormones contrôlant la résorption osseuse agissent indirectement sur l'ostéoblaste, pour favoriser la formation des ostéoclastes. On retrouve ici des hormones systémiques classiques (PTH, VitD3), des cytokines (IL-1, TNF) qui induisent (1) (initiation) dans la cellule la production de facteurs locaux (2) (IL-6, IL-11, PGE2, MCSF). Des facteurs locaux intermédiaires comme IL-6, IL-11, PGદ2 induisent l'expression du facteur RANKL, qui lui-même agit sur son récepteur RANK porté par le précurseur ostéoclastique (3). RANKL est le principal inducteur physiologique du programme de différenciation des ostéoclastes (4). La leptine (produite par le tissu adipeux) agit via l'hypothalamus et le récepteur $\beta 2 A R$ sur les ostéoblastes (adapté de [1]). 
(receptor activator of $N F-\kappa B$ ligand) dans les ostéoblastes, le principal facteur de différenciation des ostéoclastes. L'activation sympathique via la stimulation de ADR 32 stimule la phosphorylation d'ATF4, un membre de la famille des facteurs de transcription CREB/ATF, exprimé uniquement dans les ostéoblastes. La leptine exerce son effet sur l'ostéoblaste en induisant la différenciation des ostéoclastes via le système sympathique. Elle est en outre la seule hormone à avoir une influence sur les deux aspects du remodelage osseux - influence exercée dans les deux cas de façon centrale. Ajoutant à l'importance de cette régulation, les souris $A D R \beta 2^{-/-}$sont protégées de la perte osseuse induite après ovariectomie. Cela indique que l'ostéoporose met en jeu des processus centraux. Le phénotype osseux des souris $A D R \beta 2^{-/-}$ était inattendu car les souris $o b / o b$, bien qu'elles aient également une augmentation des marqueurs de formation osseuse, ont un niveau de résorption osseuse accéléré, et ce en dépit d'une activité sympathique diminuée. L'absence de perte osseuse des souris $A D R \beta 2^{-/-}$après gonadectomie était d'autant plus surprenante qu'elles devraient être une exacte phénocopie des souris ob/ob. Elles sont hypogonadiques et ont un tonus sympathique bas.

\section{La leptine diminue la résorption osseuse par la voie CART}

Cet apparent paradoxe est expliqué par le fait que la leptine exerce une autre régulation, cette fois-ci négative, sur la résorption osseuse en utilisant comme médiateur le CART (cocaine and amphetamine related transcript), dont l'expression est régulée par la leptine mais dont l'invalidation n'entraîne pas d'obésité [4]. Les souris invalidées pour le gène CART ont une masse osseuse basse liée à une augmentation marquée du nombre et de l'activité des ostéoclastes. Le phénotype osseux de souris déficientes en récepteur des mélanocortines de type 4 $\left(M C 4 R^{-/-}\right)$et de patients porteurs de mutations MC4R a aussi été étudié. L'absence de MC4R est associée à une augmentation de l'expression de CART dans l'hypothalamus. Les mutations MC4R chez l'homme ont des conséquences sur la fonction du récepteur et des patients homozygotes MC4R sont décrits $[5,6]$. Les souris $M C 4 R^{-/-}$ont une réduction du nombre d'ostéoclastes et

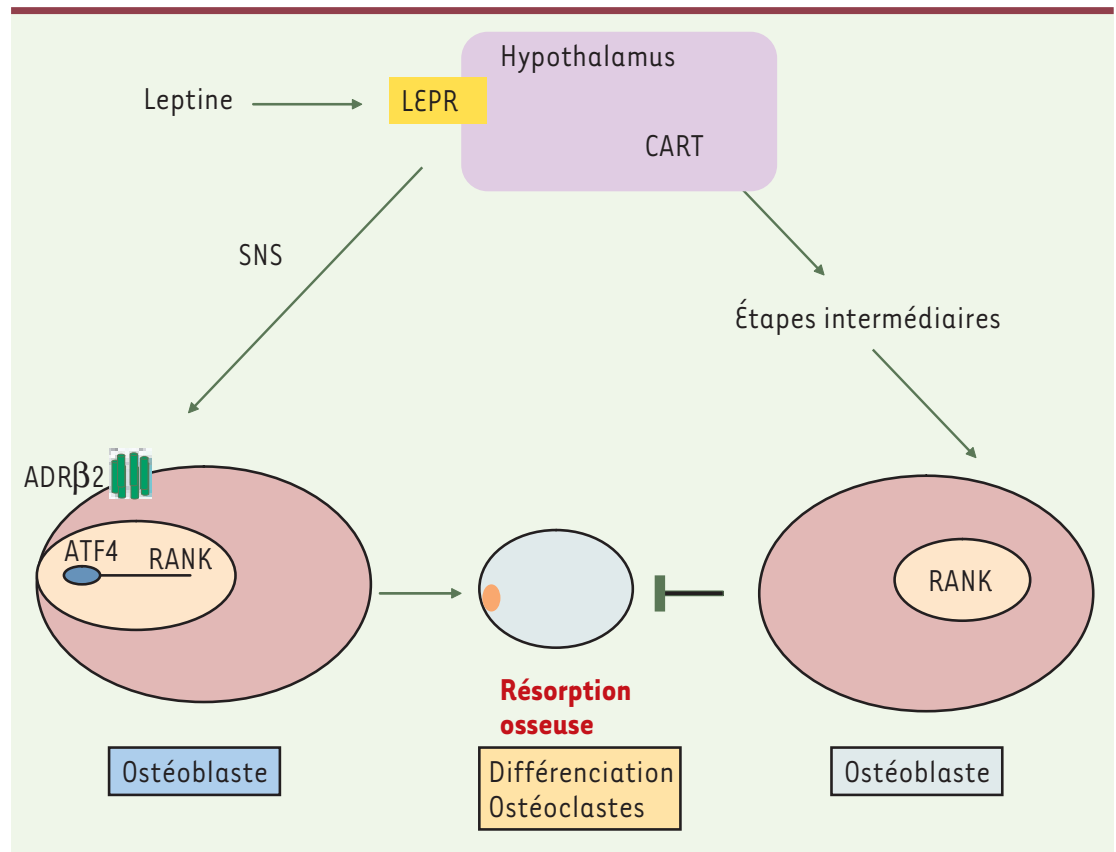

Figure 2. Modèle du contrôle central de la résorption osseuse. La leptine contrôle la résorption osseuse par deux mécanismes distincts et antagonistes : d'une part, la voie sympathique via son action sur le récepteur $\beta 2$-adrénergique et, d'autre part, via la voie CART.

les marqueurs de formation osseuse sont normaux. Chez les patients MC4R déficients, les marqueurs de formation ostéoblastique étaient diminués, suggérant une baisse des processus de résorption osseuse et expliquant une augmentation de la densité osseuse observée [6]. La leptine contrôle donc la résorption osseuse par deux mécanismes distincts et antagonistes: d'une part, la voie sympathique via son action sur le récepteur $\beta 2$ adrénergique et, d'autre part, via la voie CART (Figure 2). Ces deux voies régulent l'expression de RANKl. Un aspect remarquable de la régulation de la masse osseuse par la leptine est qu'elle utilise des médiateurs spécifiques. Les souris n'ayant pas de CART ou pas de tonus sympathique ont un poids normal et sont fertiles. Cette spécificité est une indication que le contrôle de masse osseuse est probablement une fonction ancestrale de la leptine, une hormone qui apparaît durant l'évolution avec le remodelage osseux. Chez les souris $o b / o b$, on observe donc à la fois l'effet de la carence chronique en leptine et l'effet du tonus CART altéré (levée de l'inhibition sur la résorption osseuse), expliquant ainsi le phénotype d'une augmentation des marqueurs de formation osseuse et une augmentation des marqueurs de résorption. Du fait du rôle clé du système sympathique dans ces processus, il serait intéressant de tester l'effet des $\beta$-bloquants sur le maintien de la masse osseuse. $\diamond$

Neural control of bone remodeling: crucial role of leptin

\section{RÉFÉRENCES}

1. Boissy P, Malaval L, Jurdic P. Ostéoblastes et ostéoclastes : une coopération exemplaire entre cellules mésenchymateuses et cellules hématopoïétiques. Hématologie 2000 ; 6:6-16.

2. Ducy P, Amling M, Takeda S, et al. Leptin inhibits bone formation through a hypothalamic relay: a central control of bone mass Cell 2000; $100: 197-207$

3. Elefteriou F, Ahn JD, Takeda S, et al. Leptin regulation of bone resorption by the sympathetic nervous system and CART. Nature 2005 ; $434: 514-20$

4. Kristensen P, Judge ME, Thim L, et al. Hypothalamic CART is a new anorectic peptide regulated by leptin. Nature $1998 ; 393: 72-6$

5. Vaisse C, Clément K, Durand $\varepsilon$, et al. Melanocortin-4 receptor mutations are a frequent and heterogeneous cause of morbid obesity. J Clin Invest 2000 ; 106 : 253-62.

6. Farooqi IS, O'Rahilly S. Monogenic obesity in humans. Annu Rev Med 2005 ; 56 : 443-58. 